\title{
An Investigation into the Use of 3G Mobile Communications to Provide Telehealth Services in Rural KwaZulu-Natal
}

\author{
Malcolm Clarke, $P h D,{ }^{1}$ and Maurice Mars, $M D^{2,3}$ \\ ${ }^{1}$ School of Information Systems, Computing, and Mathematics, \\ Brunel University, Uxbridge, United Kingdom. \\ ${ }^{2}$ Nelson Mandela School of Medicine, Durban, South Africa. \\ ${ }^{3}$ The University of KwaZulu-Natal, Durban, South Africa.
}

\begin{abstract}
Background: We investigated the use of third-generation (3G) mobile communications to provide telehealth services in remote health clinics in rural KwaZulu-Natal, South Africa. Materials and Methods: We specified a minimal set of services as our use case that would be representative of typical activity and to provide a baseline for analysis of network performance. Services included database access to manage chronic disease, local support and management of patients (to reduce unnecessary travel to the hospital), emergency care (up to $8 \mathrm{~h}$ for an ambulance to arrive), e-mail, access to up-todate information (Web), and teleclinics. We made site measurements at a representative set of health clinics to determine the type of coverage (general packet radio service [GPRS]/3G), its capabilities to support videoconferencing (H323 and Skype ${ }^{\mathrm{TM}}$ [Microsoft, Redmond, WA]) and audio (Skype), and throughput for transmission control protocol (TCP) to gain a measure of application performance. Results: We found that none of the remote health clinics had $3 G$ service. The GPRS service provided typical upload speed of 44 kilobits per second (Kbps) and download speed of 64 Kbps. This was not sufficient to support any form of videoconferencing. We also observed that GPRS had significant round trip time (RTT), in some cases in excess of $750 \mathrm{~ms}$, and this led to slow start-up for TCP applications. Conclusions: We found audio was always so broken as to be unusable and further observed that many applications such as Web access would fail under conditions of very high RTT. We found some health clinics were so remote that they had no mobile service. 3G, where available, had measured upload speed of $331 \mathrm{Kbps}$ and download speed of 446 Kbps and supported videoconferencing and audio at all sites, but we frequently experienced $3 G$ changing to GPRS. We conclude that mobile communications currently provide insufficient coverage and capability to provide reliable clinical services and would advocate dedicated wireless services where reliable communication is essential and use of store and forward for mobile applications.
\end{abstract}

Key words: e-health, mobile health, telecommunications, telemedicine, telehealth

\section{Introduction}

he problem of access to health services in remote locations in the developing world is well documented. ${ }^{1}$ Numerous projects have described how they have attempted to overcome the limitations of the communication infrastructure in these countries through the use of mobile communications. ${ }^{2}$ Some countries have adopted a national policy to prioritize development of the communication infrastructure in an attempt to address the problem. ${ }^{3}$ However, there are many practical problems to be overcome: communications are managed by commercial organizations, and the requirements for health may not be commercially viable; land lines are frequently stolen; communication costs in the developing world remain high compared with GDP; communications are sparse (e.g., less than $2 \%$ have a land line); populations are sparse in remote locations; distances and geographic area coverage are large; services are not available everywhere; terrain can be hilly; and funding for projects is scarce.

Health services in remote locations can differ greatly, but there is a general desire to improve the health of the citizens of a country by increasing the capability of rural health clinics and the staff. Adoption of information communication technology can enable high-quality services to be offered and staff to be supported. Computers can support access to patient databases, administration functions, communication, education, and information. Advanced functions can include supporting teleclinics (connecting a patient with a distant physician), telehealth (forwarding physiological data), and imaging. To provide high-quality services requires good communication infrastructure. Typically, this can be provided through fixed lines, but for reasons stated, this is not always possible in the remote locations of many rural health clinics, and other communication technologies must be used.

Mobile communications are often believed to offer a universal solution to these problems. We therefore undertook an investigation into the feasibility of using third-generation (3G) mobile communications to support telehealth services in rural KwaZulu-Natal, South Africa. This article reports our investigation to determine whether $3 \mathrm{G}$ could be a cost-effective alternative to terrestrial communications.

\section{Materials and Methods}

We made measurements of mobile communication service provision at a representative set of rural health clinics in KwaZulu-Natal, in order to ascertain coverage, type of service available, capability to support clinical services, and characteristics of the communication.

\section{COVERAGE}

Measurements were made at representative sites around KwaZuluNatal to determine coverage at different types of health organizations, 


\section{CLARKE AND MARS}

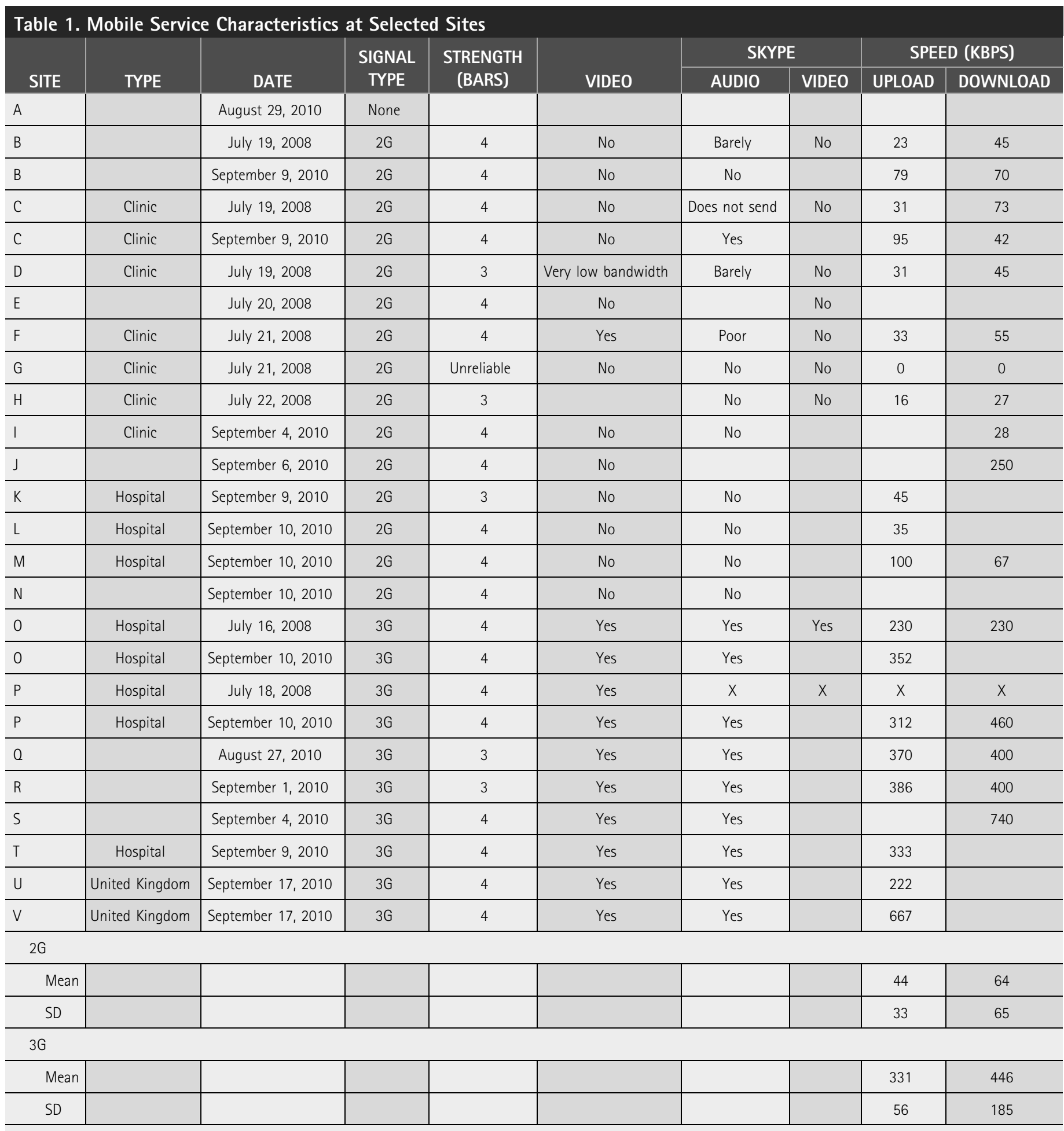

$2 \mathrm{G}$, second generation; $3 G$, third generation; $S D$, standard deviation. 
including hospitals, rural clinics, and some other selected locations. Technical performance measurements were conducted to determine the type of mobile service available and to determine the capabilities of the service at selected sites. Measurements were made during two periods: July 2008 and August 2010.We made repeated measurements at several locations to determine if the service had been upgraded over time.

\section{CAPABILITY AND CHARACTERISTICS}

A laptop was configured with a $3 \mathrm{G} /$ general packet radio service (GPRS) modem on the Vodacom mobile network in KwaZulu-Natal. Wireshark ${ }^{4}$ was used to capture and analyze network traffic to determine throughput for applications. Iperf ${ }^{5}$ was used to determine sustained throughput for transmission control protocol (TCP) traffic. As 3G and GPRS are asymmetric in bandwidth, throughput in both the up and down direction was determined. Polycom (San Jose, CA) PVX ${ }^{\mathrm{TM}}$ was used as the videoconference application (H323), with a connection being made to a Sony (Tokyo, Japan) videoconference system in the Nelson Mandela School of Medicine, Durban, South Africa. Skype ${ }^{\mathrm{TM}}$ (Microsoft, Redmond, WA) was used as an example of an Internet-based video/voice application. Skype was tested by making an audio connection to the Skype echo service. Web access to typical Internet sites was used as an example network application.

\section{Results}

Several measurements were taken at each site. Results were consistent for each site regarding the service available (3G or GPRS) and its characteristics and are presented in Table 1.

\section{COVERAGE AND PERFORMANCE}

For sites with $3 \mathrm{G}$, the results were consistent between sites and between the results from 2008 and 2010. Upload speed for the network was determined to provide a sustained throughput of around
$331 \mathrm{Kbps}$ for TCP traffic, and download speed was determined to be around $461 \mathrm{Kbps}$. This was deemed sufficient to support video services at $256 \mathrm{Kbps}$ and provide good access to Web sites that did not have significant image content.

Videoconference sessions held with the Department of Telehealth of the Medical School in Durban, using a bandwidth of $256 \mathrm{Kbps}$, were found to be consistently good. Jitter and loss for video and audio packets were consistently low, demonstrating the network is capable of sustaining video as an application. Higher connection speeds did not provide sustained good performance, with loss and jitter increasing rapidly with higher connection speeds, as is consistent with measured throughput. Access to Web services was good and consistent with measured throughput.

Coverage of 3G was limited to urban areas, business areas, or popular tourist destinations. There was no 3G coverage in any of the rural areas, unless close to a tourist destination.

We measured GPRS as a means of providing network access for rural clinics. Average upload speed was $44 \mathrm{Kbps}$, and average download speed was $64 \mathrm{Kbps}$. We noted that bandwidth was significantly improved from measurements in 2008, when these were $30 \mathrm{Kbps}$ as upload speed and $40 \mathrm{Kbps}$ as download speed. We would expect this as a result of the introduction of EDGE (2.5 generation) to the network to enhance GPRS (second generation).

Some remote clinics had no mobile service at all. These typically were in locations far from the nearest town and in radio shadow from masts due to the local terrain.

\section{EXPERIENCED PERFORMANCE}

We experienced unreliable communication and performance with Web applications in all sites. When investigated, we determined that round trip times (RTTs), as measured with ping, were around $300 \mathrm{~ms}$ for $3 \mathrm{G}$ and over $750 \mathrm{~ms}$ for GPRS. This compares with $20 \mathrm{~ms}$ for typical terrestrial networks. Several applications had timeout problems and would sever the connection. Although these speeds should

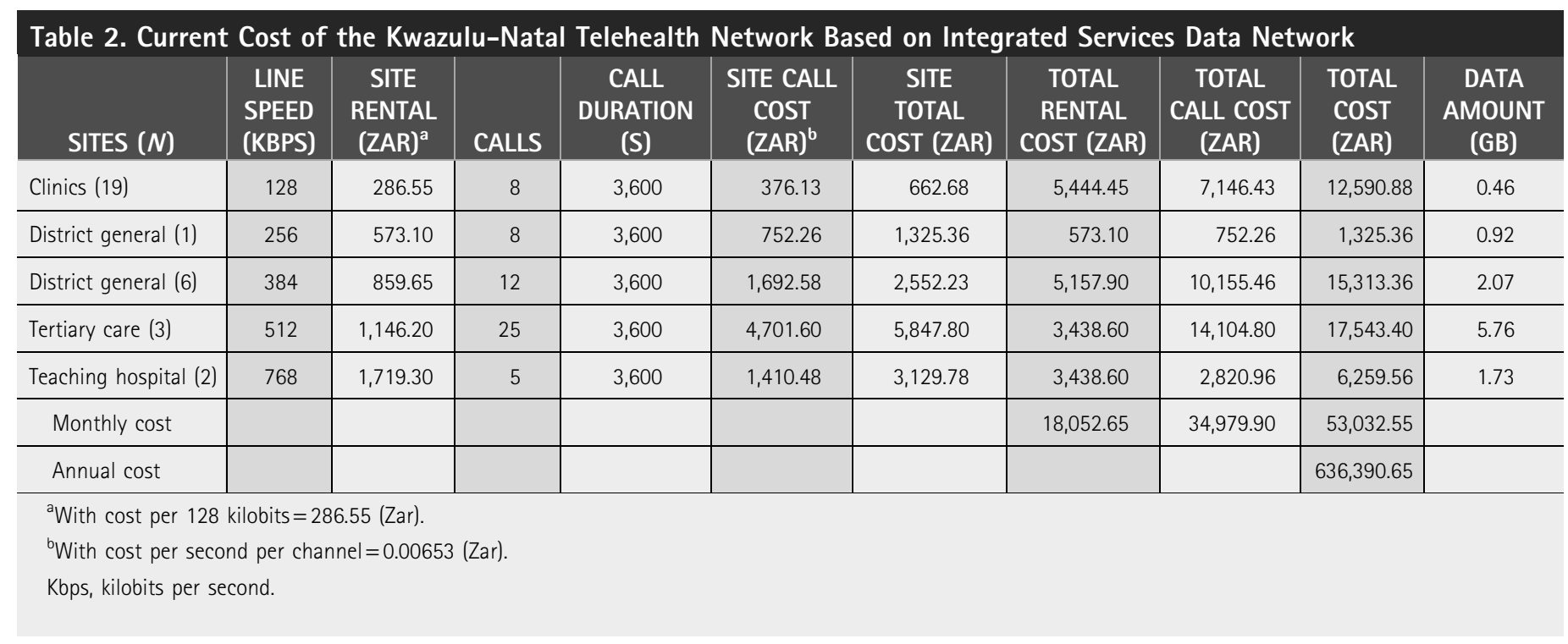




\section{CLARKE AND MARS}

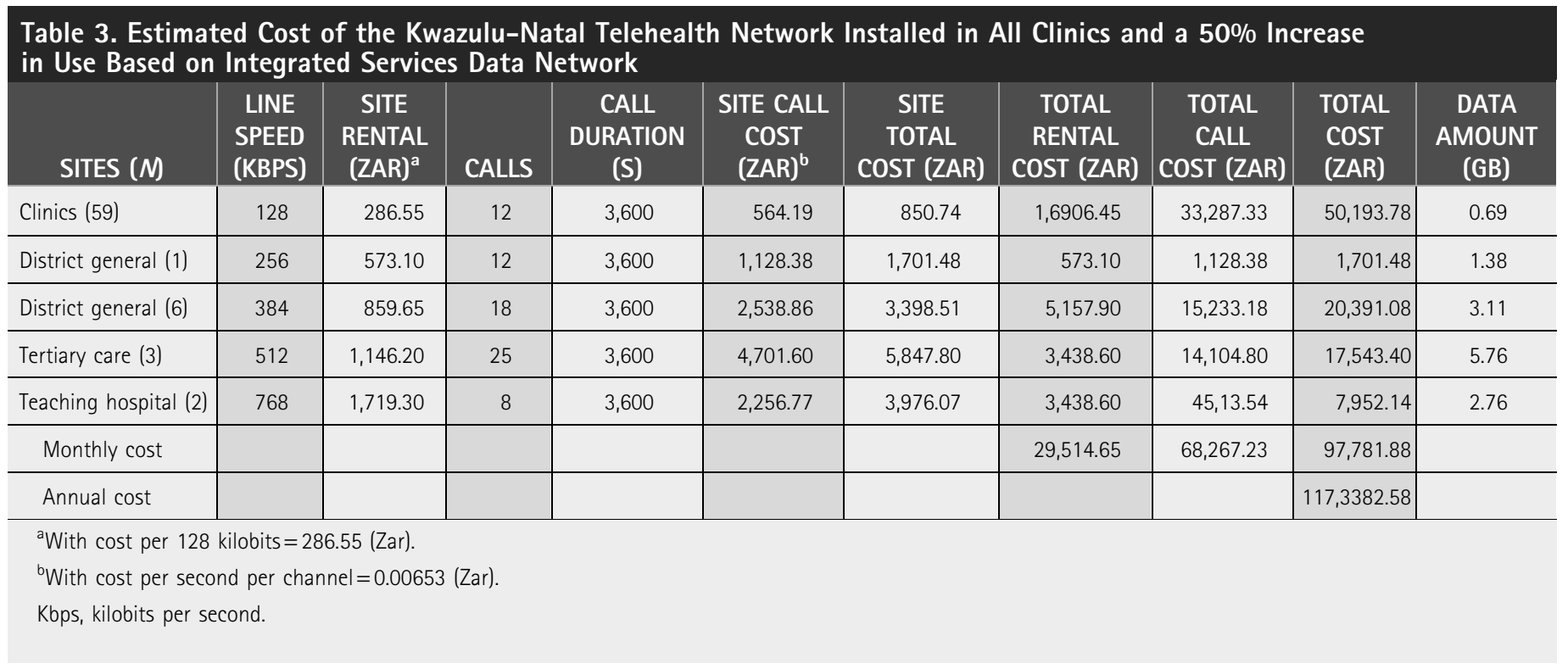

support Web applications, the large RTTs of GPRS result in poor perceived performance due to the slow start-up algorithm of TCP.

RTT does not affect video traffic as it is based on the user datagram protocol of the Internet protocol.

In areas with $3 \mathrm{G}$, we noticed that service would occasionally downgrade to GPRS for periods of time.

\section{FINANCIAL CONSIDERATIONS}

We developed a model of the KwaZulu-Natal telehealth network that is currently based on Integrated Services Data Network (ISDN) in order to compare cost with provision through other technologies. We selected dedicated wireless (microwave) links. We also considered cost assuming 50\% increased use of the network.

The first scenario (Table 1) presents the actual use of the current network based on ISDN. The second scenario (Table 2) considers extending the network so that all hospitals and clinics in the state are connected based on the same ISDN and assumes that this leads to increased use of 50\% in the district hospitals (equivalent to an increase from $2 \mathrm{~h}$ to $3 \mathrm{~h}$ of use per week).

The third scenario (Table 3) considers using fixed wireless technology (microwave link) to provide reliable network infrastructure for all sites (in practice, fixed links are already available in the large hospitals). Not only is the third scenario reliable, it can provide improved bandwidth and access at lower cost. Furthermore, all services (e.g., telephony) can be provided over the same connection, thereby providing savings from eliminating multiple connections (in some cases, satellite phone).

\section{Discussion}

We have undertaken an investigation into the feasibility of using 3G/ GPRS to support telehealth services in KwaZulu-Natal. Performance measurements confirm that $3 \mathrm{G}$ can support sustained throughputs of at least $250 \mathrm{Kbps}$ in the upload and download directions and did support good-quality videoconferencing at $256 \mathrm{Kbps}$.

However, 3G was only available in urban areas (where terrestrial communications are also available), with no coverage in rural areas. GPRS was widely available, and measured bandwidth should support e-health applications. However, experience in KwaZulu-Natal revealed excessive RTTs that caused many e-health applications to fail.

We also determined several locations with extremely poor or no mobile service.

We accept that mobile communications can be an excellent solution in rural areas, but care has to be exercised in the choice of application and implementation. Mobile communications can provide ideal solutions to mobile workers under the condition that applications can work offline in situations where there is no signal, so that the application has sufficient information to be useful and can

\begin{tabular}{|c|c|c|}
\hline SITES $(N)$ & SITE COST (ZAR) & TOTAL COST (ZAR) \\
\hline Clinics (59) & 855 & 50,445 \\
\hline District general (1) & 855 & 855 \\
\hline District general (6) & 855 & 5,130 \\
\hline Tertiary care (3) & 1,200 & 3,600 \\
\hline Teaching hospital (2) & 855 & 1,710 \\
\hline Monthly cost & & 61,740 \\
\hline Annual cost & & 740,880 \\
\hline
\end{tabular}


store data for later upload and synchronization. There are numerous examples of this approach. ${ }^{2,6}$

However, in the context of this research, telehealth was to be provided in the rural clinics and, therefore, with fixed and static installation of the communication infrastructure. We conclude that the mobile network is currently not suitable to provide the infrastructure for a telehealth network that must provide reliable health services and would advocate use of dedicated wireless links in this case. This could also lead to cost savings (Table 4).

\section{Acknowledgments}

The authors acknowledge support from the Royal Society, United Kingdom, which funded this research.

\section{Disclosure Statement}

No competing financial interests exist.

\section{REFERENCES}

1. Mars $\mathrm{M}$, et al. Telemedicine in sub-Saharan Africa. In: Telehealth in the developing world. London: Royal Society of Medicine Press, 2009:232-241.
2. Déglise $C$, Suggs LS, Odermatt P. SMS for disease control in developing countries: A systematic review of mobile health applications. J Telemed Telecare 2012;18:273-281.

3. Chanda KL, Shaw JG. The development of telehealth as a strategy to improve health care services in Zambia. Health Inform Libraries J 2010;27:133-139.

4. Available at www.wireshark.org (last accessed March 4, 2014).

5. Available at http://iperf.sourceforge.net/ (last accessed March 4, 2014).

6. Rashid AT, Elder L. Mobile phones and development: An analysis of IDRCsupported projects. Electron J Inf Syst Dev Countries 2009;36:1-16.

Address correspondence to: Malcom Clarke, PhD School of Information Systems, Computing, and Mathematics Brunel University Kingston Lane Uxbridge, Middlesex UB8 3PH United Kingdom

E-mail: malcolm.clarke@brunel.ac.uk

Received: April 25, 2014 Accepted: April 25, 2014 